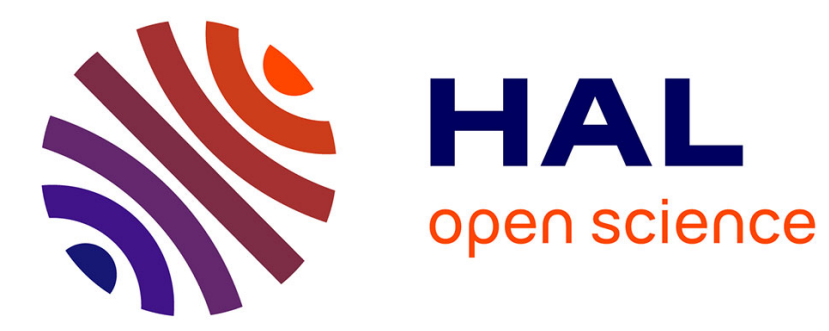

\title{
Using wrist vibrations to guide hand movement and whole body navigation
}

Anke Brock, Slim Kammoun, Marc J.-M. Macé, Christophe Jouffrais

\section{To cite this version:}

Anke Brock, Slim Kammoun, Marc J.-M. Macé, Christophe Jouffrais. Using wrist vibrations to guide hand movement and whole body navigation. i-com, 2014, Special Issue: Accessibility, 13 (3), pp. 19-28. 10.1515/icom.2014.0026 . hal-01153779

\section{HAL Id: hal-01153779 \\ https://hal.science/hal-01153779}

Submitted on 20 May 2015

HAL is a multi-disciplinary open access archive for the deposit and dissemination of scientific research documents, whether they are published or not. The documents may come from teaching and research institutions in France or abroad, or from public or private research centers.
L'archive ouverte pluridisciplinaire $\mathbf{H A L}$, est destinée au dépôt et à la diffusion de documents scientifiques de niveau recherche, publiés ou non, émanant des établissements d'enseignement et de recherche français ou étrangers, des laboratoires publics ou privés. 


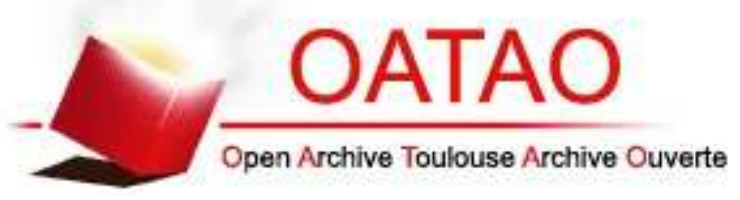

\section{Open Archive TOULOUSE Archive Ouverte (OATAO)}

OATAO is an open access repository that collects the work of Toulouse researchers and makes it freely available over the web where possible.

This is an author-deposited version published in : http://oatao.univ-toulouse.fr/ Eprints ID : 13276

To link to this article : DOI: $10.1515 /$ icom.2014.0026

URL : http://dx.doi.org/10.1515/icom.2014.0026

To cite this version : Brock, Anke and Kammoun, Slim and Macé, Marc and Jouffrais, Christophe Using wrist vibrations to guide hand movement and whole body navigation. (2014) i-com Zeitschrift für interaktive und kooperative Medien, vol. 13 (n³). pp. 19-28. ISSN 1618-162X

Any correspondance concerning this service should be sent to the repository administrator: staff-oatao@listes-diff.inp-toulouse.fr 


\section{Using wrist vibrations to guide hand movement and whole body navigation}

accessibility_visual impairment_tactons_wearables_navigation_interactive maps_virtual environment

\begin{abstract}
Summary. In the absence of vision, mobility and orientation are challenging. Audio and tactile feedback can be used to guide visually impaired people. In this paper, we present two complementary studies on the use of vibrational cues for hand guidance during the exploration of itineraries on a map, and whole body-guidance in a virtual environment. Concretely, we designed wearable Arduino bracelets integrating a vibratory motor producing multiple patterns of pulses. In a first study, this bracelet was used for guiding the hand along unknown routes on an interactive tactile map. A wizard-of-Oz study with
\end{abstract}

six blindfolded participants showed that tactons, vibrational patterns, may be more efficient than audio cues for indicating directions. In a second study, this bracelet was used by blindfolded participants to navigate in a virtual environment. The results presented here show that it is possible to significantly decrease travel distance with vibrational cues. To sum up, these preliminary but complementary studies suggest the interest of vibrational feedback in assistive technology for mobility and orientation for blind people.

\section{Introduction}

Visually impaired (VI) people face important challenges in their daily lives. Knowing one's environment, knowing where to go, and traveling autonomously are among the biggest ones. Indeed, more than half of the blind population in France faces challenges regarding mobility and orientation (C2RP, 2005). In the past years, solutions have emerged both for guiding $\mathrm{VI}$ people while traveling in an unknown environment (Roentgen et al., 2008), and for acquiring spatial knowledge with the help of accessible maps (Brock, Oriola, et al., 2013).

In the absence of vision - and hence in assistive technologies for VI people non-visual cues (mostly audio and tactile cues) are used for human-computer interaction (Brock, Kammoun, et al., 2013). The tactile modality encompasses cutaneous sensations (touching of the skin of any part of the body which remains motionless), kinesthetic sensations (perception of the relative position and movement of body parts) and haptic sensations (combination of cutaneous and kinesthetic perceptions across space and time). Tactile interfaces rely on actuators that act as force and position sources on the human skin to elicit a tactile sensation (El Saddik et al., 2011), as for instance vibro-tactile actuators. Smartphones, tablets and wearable devices are widely spreading over the market and integrate vibro-tactile actuators. They provide designers with the possibility to integrate vibrational cues in prototypes in an easy and affordable manner. In contrast to audio interfaces, tactile interfaces are silent and thus raise less privacy issues. For blind people, tactile interfaces provide the advantage of not masking audio cues that are used for orientation and navigation. It has also been shown that vibro-tactile feedback could facilitate orientation and navigation in the absence of vision (see for instance Yatani et al., 2012). However, knowledge about the effectiveness of tactile feedback has been presented sparsely and without a comprehensive understanding of which dimensions are better applicable for guidance. Thus, there still is a need for further investigation and comparison of vibro-tactile output with other output modalities.

In the current paper, we present two preliminary experiments that we conducted with the same vibrating bracelets with blindfolded sighted participants. In a first study, we showed that vibro- tactile feedback could be used to guide the hand along unknown itineraries on an audio-tactile map. This technique proved to be more efficient than auditory feedback. In a second study, we explored whether the same vibrational wrist feedback could be used for guiding an avatar along a straight line within a virtual environment. We showed that it significantly helps blindfolded users to reduce the deviation from the optimal path.

\section{Related Work: Vibro-tactile output in interactive systems}

\subsection{Vibro-tactile interaction techniques}

The tactile modality is an efficient output modality in different situations, e.g. when natural sounds are critical and should not be masked, when ambient noise level is too high, or when impairment prevents auditory-based interaction (visual impairment is sometimes associated with auditory impairment). In this paper, we focus on vibrational cues for non-visual interaction. Choi and Kuchenbecker (2013) presented a comprehensive re- 
view of vibro-tactile displays, with regard to the limits of human perception as well as technical possibilities. They described several aspects that impact the design of vibro-tactile displays. The first aspect concerns the perception thresholds in terms of intensity and frequency of vibrations. The second aspect concerns the discrimination capabilities between different vibro-tactile patterns. The third aspect concerns the temporal discrimination of cues. Human beings are able to finely discriminate tactile stimuli with rhythmic differences, i.e. changes in the amplitude over time. Finally, depending on the frequency and amplitude, the vibration can be perceived as rough or smooth.

Brewster and Brown (2004) introduced tactons, also called tactile icons. Tactons are structured, abstract messages that make use of vibro-tactile sensations to convey information. Thus they are the tactile equivalent to icons and earcons (Blattner et al., 1989). As for earcons, the message conveyed by tactons is not explicit and has to be learnt. Advantages are that tactons are quicker to perceive than braille text and that they are universal and not bound to a specific language. Numerous tactons can be robustly distinguished (Choi \& Kuchenbecker, 2013). Tactons possess different parameters that can be modified (Brewster \& Brown, 2004). These parameters are the frequency (within the range of perceivable frequencies: 20 to $1000 \mathrm{~Hz}$ ), the amplitude (i.e. intensity), the waveform and duration, as well as the rhythm.
Furthermore, Brewster and Brown suggested that the location of the actuator with regard to the body could be used for coding the tactons. Often vibration is presented at the fingertips of the user. However, other body parts can also perceive vibration and this has successfully been used in different prototypes (see for instance Pielot et al., 2009).

Tactile interaction provides an advantage over audio interaction in that it is private and does not mask surrounding sounds. Indeed, in a previous study in which feedback was provided via speech output, some blind participants described the interface as being too noisy (Brock et al., 2014). Disadvantages of vibrotactile interfaces may include "tactile fatigue" resulting from a continuous or repetitive stimulation of the receptors and a limited information bandwidth as compared to speech.

\subsection{Vibro-tactile feedback for navigation and guidance}

Different studies have shown the usability of tactile cues for presenting directions by mapping them onto body locations. Tsukada \& Yasumura (2004) proposed a belt with spatialized vibrations. Eight vibromotors were attached to the belt, so that the tactile cues were equally distributed around the user's waist. Directions were indicated by activating the corresponding motors. A similar belt has proved efficient to decrease the number of errors during map-based wayfinding (Pielot et al., 2009). In another study, Pielot et al. (2011) proposed the TactileCompass. This device with one vibrational motor provided information on directions on a $360^{\circ}$ circle by varying the length of two subsequent pulses within one pattern. Distances were also provided through a variation in the pause between subsequent patterns, with short pauses corresponding to short distances. Yatani et al. (2012) equipped a smartphone with nine vibromotors. Spatialized vibrations were then used for augmenting an interactive map application. Kammoun, Jouffrais, et al. (2012) demonstrated that vibrational wristbands could be used for guiding blind people in a real environment. Finally, Weber et al. (2011) observed that vibro-tactile feedback issued from a wrist bracelet was advantageous over verbal feedback for indicating rotations.

\section{Designing the vibro- tactile bracelet}

In the past years, wearable devices have emerged and start to be frequently used in different contexts. For instance bracelets, such as the Jawbone Up, or smartwatches allow measuring heart rate, number of footsteps or sleep rhythm with the aim of contributing to a healthier life. These devices can have different forms and may be integrated into clothing, gloves, glasses, jewelry, etc. (Tsukada \& Yasumura, 2004).

In our studies, we used two wristbands with vibro-motors (see Figure 1).
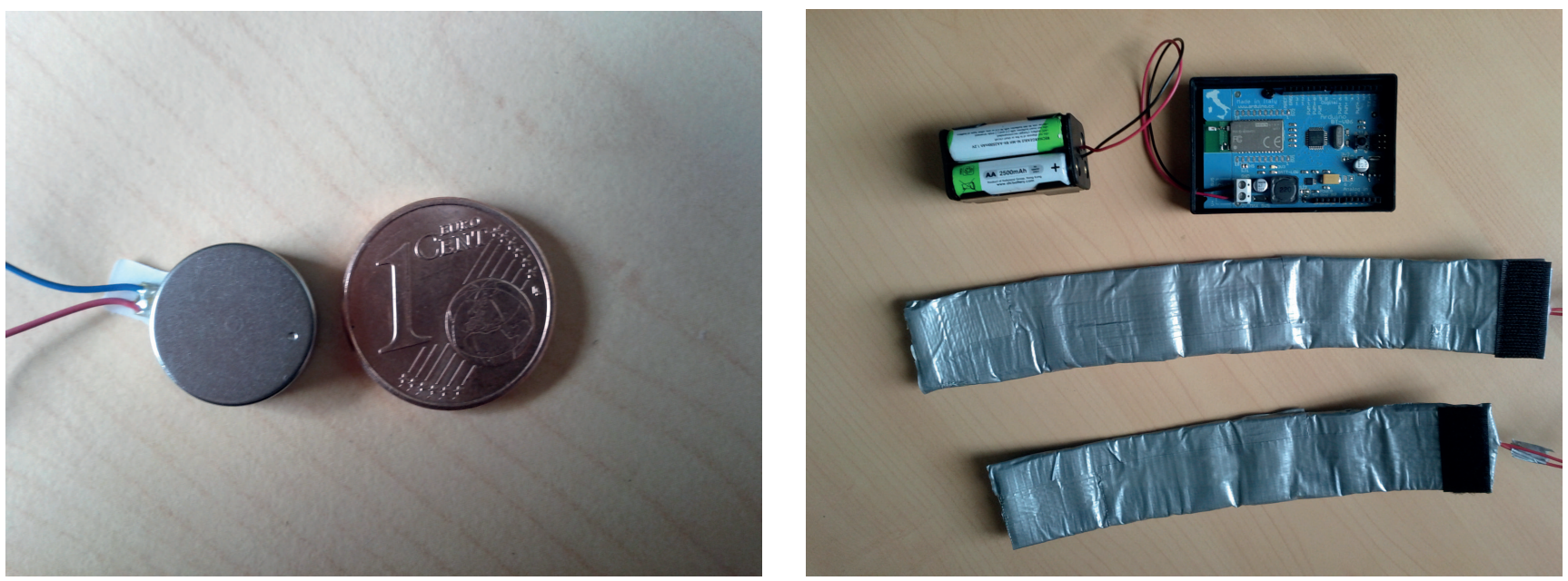

Figure 1: Photograph of the vibrating wristbands. (a) The vibrating motor. (b) The haptic bracelets with Arduino board 
These wristbands have been handmade in a prior project (Kammoun, Jouffrais, et al., 2012). Each band contained one vibration motor VPM2 (Solarbotics, Calgary, Canada). The tactile interface was programmable through an Arduino Bluetooth board and vibrational patterns were defined and uploaded on the board. The bands provided the possibility to control frequency, duration and interval between vibrational stimuli for each wristband. We also developed a smartphone application to trigger vibration signals via Bluetooth.

\section{First Study: Wrist Vibration for Route Learning on an Interactive Map}

\subsection{Related Work: Learning Routes on Interactive Maps for Visually Impaired People}

In the last 25 years, interactive technology was used to make geographic maps accessible to $\mathrm{VI}$ people (Brock, Oriola, et al., 2013). Unfortunately, current interactive maps for $\mathrm{VI}$ people are often limited to basic functionality such as providing names of streets and POI, even though the technology would allow for more advanced interactions. For instance, it would be advantageous to provide the possibility of learning routes. Route knowledge is important for autonomous navigation. It is also the basis for acquiring more flexible configurational knowledge (Thorndyke \& Hayes-Roth, 1982). Furthermore, choosing routes is critical for $\mathrm{VI}$ people as their configuration - the number of crossings, obstacles, etc. - affects safety (Williams et al., 2013). To our knowledge, few studies so far have investigated the possibility to represent route guidance on interactive maps for $\mathrm{VI}$ people. Most of these studies used audio output for guidance. For example, in the prototype by Strothotte et al. (1996) the user's finger exploring the map was guided on the route via audio cues. Pitch and balance conveyed the distance of the finger from the route.
Similarly, the TimbreMap provided a "line hinting mode" in which the user's finger was guided with stereo audio feedback (Su et al., 2010). The feedback faded out when the user's finger left the path. In the case of the One Octave Scale Interface, the user's finger was guided with musical feedback, more precisely by playing the notes of an octave along the different segments of a route (Yairi et al., 2008). Hamid \& Edwards (2013) investigated a different approach. Their prototype was composed of a multi-touch screen with raised-line overlay and audio output. The map was attached to a turntable, and in contrast with other prototypes, users could turn the map in order to adapt the map representation to their current egocentric perspective. Despite the fact that vibrational feedback has successfully been used in interactive assistive technology, it has not yet been used to provide feedback on routes in interactive maps for blind people. Consequently we address this in the current study.

\subsection{Designing Non-Visual Interaction for Route Learning on Tactile Maps}

In this preliminary study, we compared different interaction techniques for guiding a user's finger on an itinerary while exploring an interactive map based on a raised-line map overlay on a multi-touch screen (Brock et al., 2014). While the user could feel the relief of the map, the interaction technique indicated which direction to follow at the next intersection. As this study was only about exploring itineraries based on audio and tactile guidance, no information on other map elements was given. We compared an interaction technique based on wrist vibration with three existing audio-based interaction techniques.

In the first audio-based interaction technique, the user was guided with verbal description, as in a regular navigation system ("turn left at the next crossing", etc.). In case nothing was announced, the user was supposed to continue straight. We called this technique "Guided Directions" after a similar technique proposed by Kane et al. (2011). The second audio-based technique was based on the clock face method. As an example, "noon" would indicate to go straight ahead, "three o'clock" to turn eastward, etc. This idea was inspired by the fact that some blind people are used to the clock face method for orientation in a real environment. We referred to this as the "Clock Face" technique. The third audio-based technique used a musical metaphor as proposed by Yairi et al. (2008). Following the original proposition, the "One Octave Scale" did not announce the direction to follow at the next intersection. This technique thus differed from the others because, at each crossing, the user had to test all possible directions. Wrong turns were announced with the A note within the octave below. The last interaction technique was tactile and relied on the vibrating wristband. We designed tactons similar to those of Pielot et al. (2011): a short vibration followed by a long vibration indicated to turn left, a long vibration followed by a short vibration indicated to turn right, and three short vibrations indicated that a wrong turn had been taken. In case there was no indication the user had to continue straight. We called this technique "Vibrating wristband".

\subsection{Prototyping and Evaluation}

In a first step, we evaluated the interaction techniques with a Wizard of $\mathrm{Oz}$ simulation (Kelley, 1984). By doing so, we wanted to ensure that the techniques were understandable and usable before implementing them. Furthermore, the insight gained during this step helped to improve the interaction techniques.

\section{Map Drawing}

This prototype included an A3-format raised-line drawing of a street network, without providing any knowledge about names of geographical elements. We drew the streets as single lines because they are easier to follow with a finger than double lines (Tatham, 1991). We based the drawing on the road network of the city center of Lille, a city in the North of France. This road network was interesting as it was quite complex and with numerous different crossing angles. 


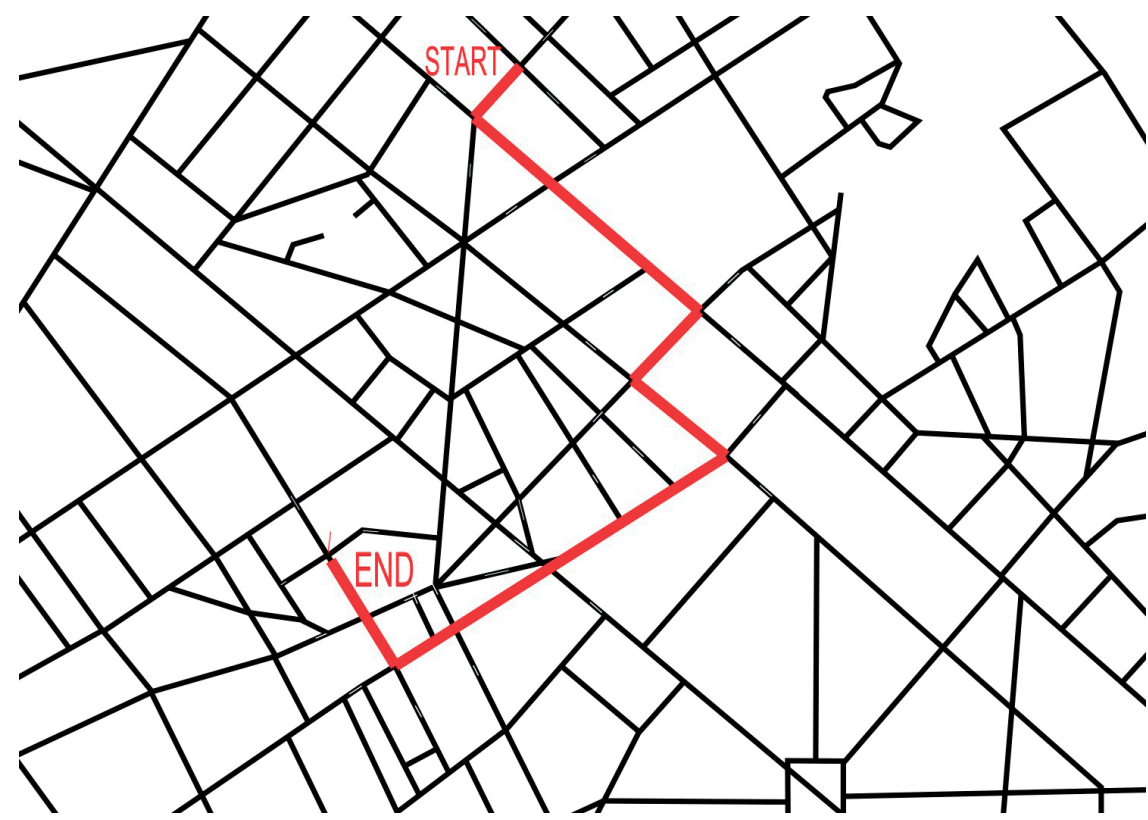

Figure 2: Example of an itinerary on the map of Lille.

In addition, given the distance between Lille and Toulouse (where our participants live), we assumed that participants did not have prior knowledge of the road network or would not recognize it. We prepared four different itineraries with a similar level of difficulty. All consisted of six segments with either two turns right and three turns left or vice versa (see Figure 2).

\section{Simulation of Output Modalities}

During the Wizard of Oz study, all interaction techniques were simulated by a human experimenter. The guidance techniques "Guided Directions" and "Clock Face" both relied on verbal output. The experimenter observed the user exploring the map and provided speech output in reaction to the exploratory movements. In order to make the simulation as realistic as possible, i.e. to avoid the user recognizing that the output was simulated, the user was wearing headphones. The experimenter used a microphone and Audacity software (http://audacity. sourceforge.net/) with the live output routed to the user's headphones. For the "Guided Directions" technique, the experimenter announced "right" or "left" shortly before each crossing. For the "Clock Face" technique, the experimenter announced the clock hour before each crossing. For both techniques, a wrong turn was also indicated verbally ("wrong way"). For the "One Octave Scale" technique, we provided musical output with a virtual midi piano. However, simulating the output proved tricky. Indeed, notes on the musical scale had to be emitted in proportion with the distance that the finger had "travelled" on the explored segment. Therefore the speed of the output had to be adapted to the speed of the finger. In order to help the experimenter play the notes at the right time, we marked the notes on the map next to each segment. Finally for the "Vibrating wristband" technique, we used a smartphone application that sends commands to the wristband via Bluetooth.

\subsection{Protocol}

\section{Participants}

Six blindfolded university students $(5 \mathrm{~m}$, 1f) participated in the experiment. Age varied between 21 and 23 with a mean of 21.8 (SD = 1.0). The female participant was the only left-handed subject. All participants provided written consent for participating in the study.

\section{Procedure}

During the experiment, users evaluated the four techniques in individual sessions. Video was recorded with the explicit agreement of the participants. At the beginning of the experiment, participants were informed about the aim of the study, i.e. testing different applications for guiding the hand along an itinerary on an interactive map. As a motivation, the experimenter introduced a scenario in which users had to prepare a holiday within an unknown city. As the blindfolded sighted participants were not used to tactile map reading, they were first allowed to familiarize with the raised-line map. They did not get any cue about the map content or itineraries, but if required, they were assisted to follow the raised lines. Once the participants felt comfortable with the map representation, the experimenter presented the task which consisted in following the guidance instructions and memorizing the itinerary. Users were informed that they would have to reproduce the itineraries afterward. Every user tested four conditions, corresponding to the four interaction techniques, in a counterbalanced order. For each condition, the experimenter first described the technique. Then, the user was allowed to follow the route twice in order to memorize it. After the route learning phase, the user answered a SUS questionnaire (Brooke, 1996) and gave qualitative feedback. The same procedure was reproduced for the three other conditions.

\section{Observed Variables}

The independent variable in our study was the type of interaction technique, which was designed as a within-participant factor. As the four routes possessed an equal number of segments and turns, we did not expect an impact of the different itineraries on the results. Nevertheless, the different interaction techniques were crossed with the four different routes. The order of presentation was randomized to prevent learning or fatigue effects. We measured the three components of usability of the interaction techniques: efficiency, effectiveness and satisfaction (ISO, 2010). Efficiency corresponded to the time elapsed between the beginning and the end of a route exploration. Effectiveness was determined as the number of errors. An error was counted when the participant was not following the instructions of the application (for instance turning left when right was indicated). Obvi- 
ously, there is a difference between One Octave Scale and the other techniques, as One Octave Scale did not indicate in which direction to turn. For this technique, an error was counted only when the participant persisted in a direction after the "wrong turn" sound was emitted. Finally, satisfaction was measured with the SUS questionnaire translated into French.

\subsection{Results}

Our aim was to test whether the tactile interaction technique differed from the auditory techniques in exploration time, number of wrong turns or satisfaction. An alpha level of .05 was used for statistical significance in every test.

Exploration time was measured for the first and the second exploration. The time values for the second exploration were normally distributed (Shapiro-Wilk $W=0.95, p=.29$ ). Accordingly, they were compared across type of interaction technique in an analysis of variance (ANOVA). The effect was not significant $(F(3,15)=2.6, p=.09)$ but a tendency emerged with the "Vibrating wristbands" being the quickest and the "Guided Directions" being the slowest technique (see Figure 3a).

Errors were not normally distributed. Therefore all errors were analyzed in a Friedman test across type of interaction technique. The number of errors for both explorations taken together was significantly different across the interaction techniques $(X 2(12)=13.97, p=.03)$. Techniques ranked in the order from the least to the most erroneous were: One Octave Scale, Vibrating Bracelets, Clock Face and Guided Directions (see Figure $3 b)$. Pairwise Wilcoxon tests with Bonferroni correction revealed that only the difference between One Octave Scale and Guided Directions was significant $(\mathrm{N}=11, \mathrm{Z}=2.93, \mathrm{p}=.003$ ).

Satisfaction was measured with the SUS questionnaire. The results of the SUS were not normally distributed (Shapiro-Wilk $W=0.91, p=.04$ ). Therefore the values were compared across type of interaction technique in a Friedman test. The result was not significant (X2(6) $=6.41, p=.09$ ). However a tendency emerged with the One Octave Scale receiving the best satisfaction values, followed by the Vibrating wristbands, the Guided Directions and finally the Clock Face (see Figure 4). We also asked participants to classify the four techniques according to their preference. The One Octave Scale was ranked five out of six times as the favorite technique. One participant liked the Guided Directions most, and one of the participants that had stated a preference for using the One Octave Scale said that the Guided Directions were better for memorization. Three participants stated that they least liked the Clock Face and two participants stated that they least like the Guided Directions.
One participant did not have a technique that he disliked most. Unexpectedly the vibrational feedback did not result in any positive or negative reactions.

Qualitative results revealed that three users would like to test two wristbands instead of one. However in our pretests we had observed better results with only one bracelet. Another participant suggested a different coding for the tactons (one long: turn right; two short: turn left; three short: wrong turn). In addition, one user suggested adding a signal for "continue straight". Several users stated that they needed to concentrate more during this technique than during the other. One user underlined that the technique demanded a higher concentration, and hence resulted in less errors. Finally, some users suggested coupling the One Octave Scale with direction information concerning the next crossing.

\subsection{Discussion and conclusions for future studies}

The results of this study need to be regarded with caution. Due to the low number of participants and trials, the statistical significance of this study is limited. Furthermore, this study was done with blindfolded sighted people, and tests with VI users will be needed as they might present different preferences and capabilities. Finally, the study was a
12

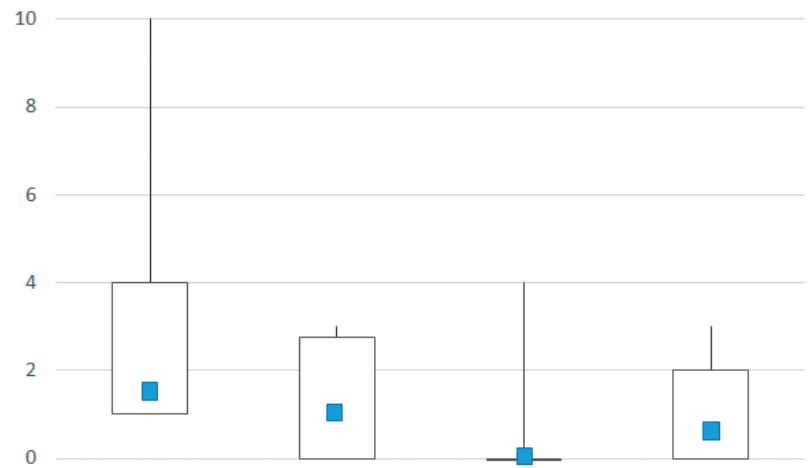

$-2$

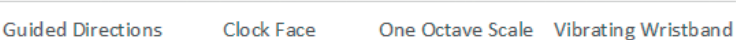

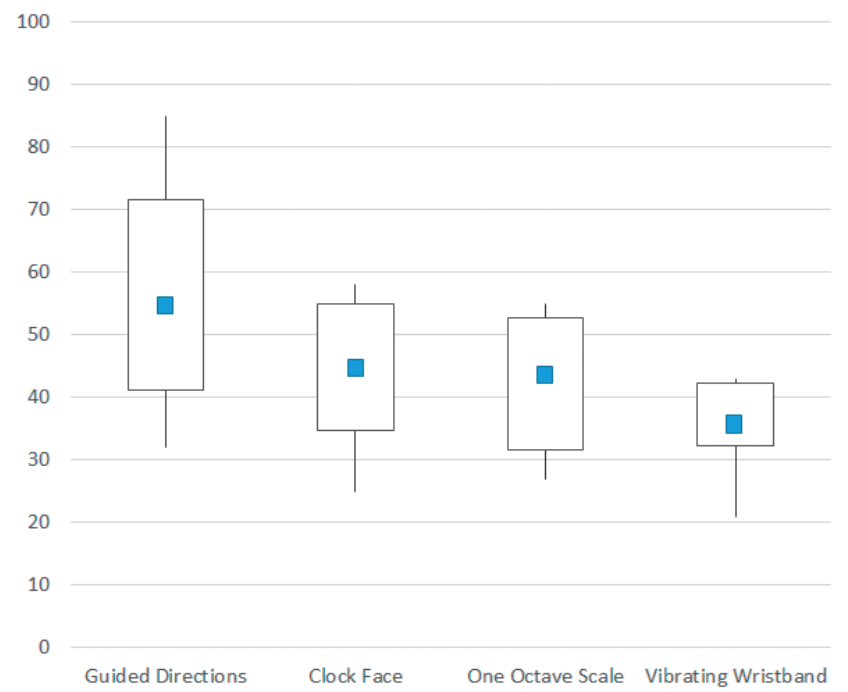

Figure 3: (a) Time of exploration. A tendency emerged with the "Vibrational wristband" being the quickest and the "Guided Directions" being the slowest technique. (b) Error values taken together for both explorations. The difference between Guided Directions and One Octave Scale was significant. 


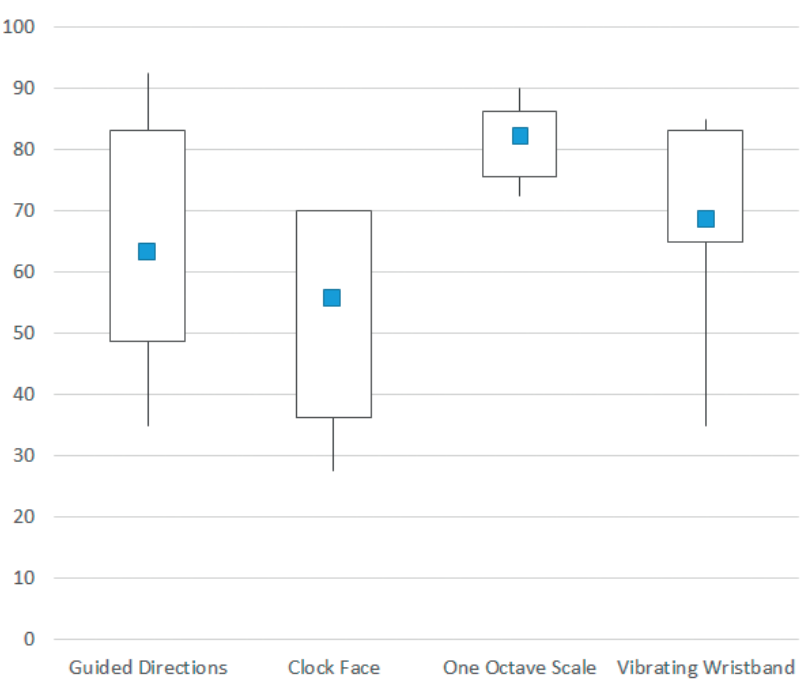

Figure 4: Satisfaction scores. A tendency emerged with the One Octave Scale receiving the best satisfaction values, followed by the Vibrating wristbands.

Wizard-of-Oz simulation and it is possible that results would differ with a high fidelity prototype. Furthermore, it would be very interesting to assess the quality of the cognitive maps resulting from route learning.

Nevertheless this study allowed us to get first insights in the differences between tactile and auditory guidance when learning routes on a map. Indeed, in the present study, the use of tactons proved to be efficient. For the second exploration trial, there was a tendency for the Vibrating wristbands to be the quickest interaction technique. The data suggested a learning effect between the first and the second exploration. Indeed, understanding tactons is not innate and has to be learnt (Brewster \& Brown, 2004). Yet, our results suggest that in case of a small number of patterns that are easy to distinguish, tactons can become an efficient means of interaction.

The positive feedback for the One Octave Scale demonstrated that users appreciated the distance information provided by this technique. It might therefore be interesting to include distance information in the other interaction techniques. As suggested by Pielot et al. (2011) for the tactile feedback, this might be done by altering the pauses between pulses (closer $=$ shorter pauses). Further studies would be needed to determine how vibrotactile patterns can represent distance information.

\section{Second Study: Wrist Vibration for Assisting Navigation in a Virtual Environment}

Virtual simulators have been developed to increase spatial cognitive abilities (Mereu \& Kazman, 1996), and to provide VI people with a tool to safely explore and learn about new spaces on their own (Schloerb et al., 2010). Generally, these systems are designed to allow VI users to explore virtual representations of real or abstract (e.g. labyrinth) spaces, as well as to interact with objects within these spaces (Sánchez $\&$ Hassler, 2006). Tactile feedback has been used within virtual environments designed to support orientation and mobility training (Schloerb et al., 2010). In this study we explored the usability of the vibro-tactile bracelets presented above to help VI users minimize the travelled distance when navigating in a virtual environment (VE). The experiments were conducted in an adapted VE called SIMU4NAV (Kammoun, Macé, \& Jouffrais, 2012).

\subsection{SIMU4NAV as a test environment}

SIMU4NAV is a simulator developed in the context of the NAVIG project (Katz et al., 2012). It is a multimodal VE facilitating the design and evaluation of electronic orientation aids (EOA). It allows systematic evaluations with blind or blindfolded users in controlled conditions before onsite implementation. The platform presents two distinct modes: a Control mode and an Exploration mode. The Control mode is used by designers, researchers, and orientation and mobility instructors for blind people, and allows the creation and modification of VEs. A key feature of the Control mode is the ability to import an XML file from Open Street Map to create a new 3D virtual map and to manually or automatically select a path between two points of this map. This makes it easy to import maps of existing places. The Control mode also includes a "feedback editor" to assign arbitrary tactile \& auditory feedback to any event in the VE. The Evaluation mode allows researchers and Orientation and Mobility instructors to record and replay the events and users' behavior.

During a session, the system logs in a text file with the information concerning the interaction (keystrokes, joystick, audio, haptic stimuli), as well as the avatar position, orientation and speed. Two observations that we made on different blind pedestrians using EOAs were modeled to improve the truthfulness of the simulator. First, it has been shown that in the absence of vision, pedestrians who intend to move along a straight path typically deviate (Souman et al., 2009). An adjustable pseudo-random drift has been added to the avatar's displacement to simulate this behavior. Second, EOAs usually rely on GNSS devices that are prone to positioning errors. An adjustable pseudorandom error was added to the location of the avatar in the VE. The platform was implemented in $\mathrm{C}++$ and the rendering was performed with the OGRE3D engine (http://www.ogre3d.org/).

\subsection{Feedback settings}

As previously mentioned, we implemented a flexible feedback editor to add simple or composite feedback according to actions done by the user in the VE (e.g. collision, walking). For this experiment, we first placed a footstep feedback, i.e. audio cues imitating footsteps, which were adjusted to walking speed. We 
then added feedback to render direction instructions, encountered obstacles and spatial configuration information with TTS and 2D/3D sounds on the audio channel. Virtual sounds positioned at the next crossing were used to provide the user with directional information. Spatialized TTS was also used to indicate the location of environmental features. We used a cross-platform 3D audio API (OpenAL) to appropriately display 3D spatialized sounds and TTS via the headphones.

We also provided tactile cues via the vibrating wristband prototype described above (Figure 1). For this experiment, we used two bracelets, each one equipped with one vibration actuator. The frequen$c y$, duration and interval between the vibro-tactile stimuli were controlled via an Arduino board connected by Bluetooth.

In this study, vibrations were used to keep the walking subject straight on the planned itinerary. A corridor of $2 \mathrm{~m}$ width had been defined around the selected route ( $1 \mathrm{~m}$ on each side). As long as the traveler kept walking within the defined corridor, there was no interruption in the overall guidance process, and planned directional instructions were appropriately provided. When substantial veering occurred, the traveler walked out of the corridor boundaries, and a vibration was triggered to get him back on the path. The same pattern (a continuous 100 ms vibration) was used either on the right or left wristband. A vibration on the right (respectively on the left) indicated that a virtual barrier had been hit and that the traveler had to correct his path back to the left (respectively to the right).

\subsection{Protocol}

\section{Participants}

Sixteen blindfolded subjects participated in this preliminary experiment $(14 \mathrm{~m}, 2$ f). Age varied between 22 and 48, with a mean of 28.7 years $(S D=6.7)$. All participants had normal hearing. They were informed about the experiment and provided written consent for participating in the study.

\section{Procedure}

Four pre-programmed routes were generated within SIMU4NAV. Each route was $225 \mathrm{~m}$ long and composed by the same number of turn angles $\left(1 * 0^{\circ}(=\right.$ no turn), $1 * 30^{\circ}, 2 * 60^{\circ}, 2 * 90^{\circ}, 2 * 120^{\circ}$ and $\left.1 * 150^{\circ}\right)$ and sections length $(1 * 40 \mathrm{~m}$, $5 * 25 \mathrm{~m}$, and $4 * 15 \mathrm{~m}$ ). Routes of equivalent complexity were generated by randomly connecting the 10 sections and 9 angles to ensure that learning would not affect the results.

The participants were blindfolded, seated in front of the desk and keyboard, and equipped with headphones. They were wearing two bracelets, one on the left and one on the right wrist. Previous studies have shown that bonephones can be successfully used in navigation tasks (Walker \& Lindsay, 2005). They present the advantage of not blocking environmental audio cues that are essential for blind people's orientation. Yet, as SIMU4NAV was used in a controlled indoor environment, we decided to use regular headphones.

Participants could move within the VE using the keyboard's arrow keys. During a session, each blindfolded subject explored a total of four different routes. In the control condition, users were guided by a virtual 3D sound coming from the next point to reach along the itinerary. Each time a waypoint was reached, the corresponding virtual sound was switched off, and the next waypoint was sonified. In the test condition, in addition to the virtual sound guidance, vibrations were triggered when the subjects deviated too much from the planned itinerary, i.e. when walking out of the corridor. All subjects' routes were logged in a text file and then analyzed.

\section{Observed Variables}

The observed variable in our study was the distance traveled by each participant within the VE. The four itineraries had the same length and the same complexity with an equal number of segments and turns, so we did not expect an effect of the itineraries on the results.

\subsection{Results}

Our aim was to test whether the wrists vibration decreased the traveled distance as compared to the audio waypoint guidance only. Figure 5 shows that the average deviation (16 subjects) from the optimal itinerary was significantly decreased with the bracelets (Anova: $F(1,62)=8.43, p=.005)$, which confirms that tactile feedback is effective to keep blindfolded travelers on a succession of straight paths.

\subsection{Discussion and conclusions for future studies}

The results presented here show that it is possible to significantly decrease the distance travelled out of a safely determined itinerary when using wrist bracelets compared to auditory feedback. This is an important finding as according to Golledge (1995), distance and time spent on route are the first criteria considered by the visually impaired people when choosing a path. Yet, the results of this study need to be regarded with caution.

\section{Distance of Deviation}

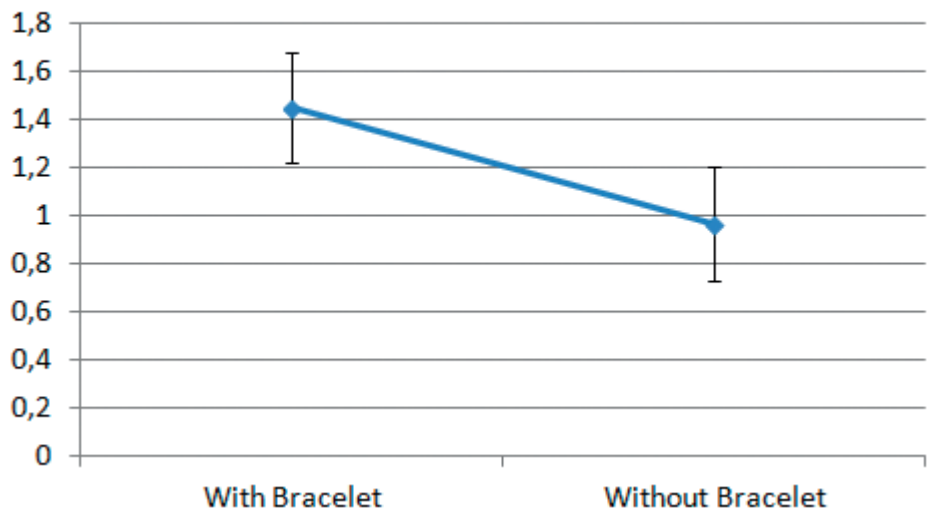

Figure 5: Average (16 subjects) travel distance out of corridor with/without vibrating bracelets. 
This study was done with blindfolded sighted people, and needs to be tested with visually impaired users as they might present different behavior. Additional evaluation could also be performed in different mobility situations; the most important point would be to check that using a guide-dog or a long cane does not impact results.

As future work, we aim at using different vibration metaphors. We could try to use a single bracelet with two different patterns for example, to indicate right or left directions as in the first study presented here.

\section{Conclusion and Perspectives}

During the last few years, several approaches have been developed to present non-visual information in the context of orientation and navigation. However, they can be seen as isolated contributions in a partly unexplored design space. Particularly, knowledge about the effectiveness of tactile feedback has been presented sparsely although it is highly relevant for blind people to provide feedback without reducing their auditory perception of the environment. Also, in comparison with audio output, vibrotactile feedback guarantees privacy and data protection and is also available for people with hearing loss.

In this paper, we present an Arduino wearable prototype using two wrist bracelets, each one equipped with a vibration actuator. This design allows to easily provide users with vibro-tactile instructions and feedback. The stimulation can be controlled in terms of frequency, duration and interval between vibrations. We used this set up in two complementary studies on the use of vibrational cues for hand guidance during map exploration, and whole body-guidance during mobility in a virtual environment. In a first Wizard-of-Oz study, we showed that vibrotactile feedback could be used for indicating directions on a tactile map. After a learning phase that is necessary to get used to the tactons, it might even be quicker to use vibrotactile than auditory feedback. In a second study, we showed that vibrotactile feedback could be used to assist blindfolded users to keep straight directions and reduce the deviation from the optimal path in a virtual environment. These two preliminary studies are complementary as they show that vibrotactile feedback could be used for indicating directions as well as for providing correctional feedback. Also they suggest that vibrotactile feedback can be useful for preparation in advance of travel as well as during mobility. Obviously, as the participants in our studies were blindfolded sighted people, these findings need to be validated with blind participants.

To conclude, both studies suggest that adding vibrational feedback to assistive technology for mobility and orientation for the blind is promising and should be systematically considered. We believe that in the future this interaction technique might be largely used, as wearable devices (e.g. smartwatches) and mobile devices (e.g. mobile phones) bring the technical possibility of easily providing vibrotactile feedback.

\section{Acknowledgments}

First, we thank the participants in our studies. Second, we would like to thank our colleagues Philippe Truillet, Bernard Oriola and undergraduate students Sophien Razali and Remy Marquerie for their help in these studies. This work was partially supported by the French $\mathrm{Na}$ tional Research Agency (ANR) through TecSan program (project NAVIG ANR08TECS-011).

\section{Bibliography}

Blattner, M., Sumikawa, D., \& Greenberg, R. J. (1989). Earcons and Icons: Their structure and common design principles. HumanComputer Interaction, 4(1), 11-44.

Brewster, S., \& Brown, L. M. (2004). Tactons: structured tactile messages for non-visual information display. In AUIC '04 Proceedings of the fifth conference on Australasian user interface (pp. 15-23). Australian Computer Society, Inc.

Brock, A. M., Kammoun, S., Nicolau, H., Guerreiro, T., Kane, S. K., \& Jouffrais, C. (2013). SIG: NVI (Non-Visual Interaction). In CHI '13 Extended Abstracts on Human Factors in Computing Systems (pp. 2513-2516). New York, New York, USA: ACM Press.

Brock, A. M., Oriola, B., Truillet, P., Jouffrais, C., \& Picard, D. (2013). Map design for visually impaired people: past, present, and future research. In B. Darras \& D. Valente (Eds.), MEI (Vol. 36, pp. 117-129). I'Harmattan.

Brock, A. M., Truillet, P., Oriola, B., Picard, D., \& Jouffrais, C. (2014). Interactivity Improves Usability of Geographic Maps for Visually Impaired People. Human-Computer Interaction.

Brooke, J. (1996). SUS: A "quick and dirty" usability scale. In P. W. Jordan, B. Thomas, B. A. Weerdmeester, \& I. L. McClelland (Eds.), Usability evaluation in industry (pp. 189-194). London, UK: Taylor \& Francis.

C2RP. (2005). Déficience Visuelle - Etudes et Résultats (pp. 1-14). Lille, France.

Choi, S., \& Kuchenbecker, K. J. (2013). Vibrotactile Display: Perception, Technology, and Applications. Proceedings of the IEEE, 101(9), 2093-2104.

El Saddik, A., Orozco, M., Eid, M., \& Cha, J. (2011). Haptics Technologies - Bringing Touch to Multimedia (p. 218). Berlin Heidelberg: Springer.

Golledge, R. (1995). Path selection and route preference in human navigation: A progress report.

Hamid, N. N. A., \& Edwards, A. D. N. (2013). Facilitating route learning using interactive audio-tactile maps for blind and visually impaired people. In CHI EA '13 Extended Abstracts on Human Factors in Computing Systems (pp. 37-42). New York, New York, USA: ACM Press.

ISO. (2010). ISO 9241-210:2010: Ergonomics of human-system interaction - part 210: human-centred design for interactive systems. Technical Specification International Organisation for Standardisation. Switzerland: ISO - International Organization for Standardization.

Kammoun, S., Jouffrais, C., Guerreiro, T., Nicolau, H., \& Jorge, J. (2012). Guiding Blind People with Haptic Feedback. In Frontiers in Accessibility for Pervasive Computing (Pervasive 2012). Newcastle, UK,

Kammoun, S., Macé, M. J.-M., \& Jouffrais, C. (2012). Multimodal Virtual Environment Subserving the Design of Electronic Orientation Aids for the Blind. In Proceedings of the 2012 ACM symposium on Virtual reality software and technology (pp. 189-190). ACM Press.

Kane, S. K., Ringel Morris, M., Perkins, A. Z., Wigdor, D., Ladner, R. E., \& Wobbrock, J. O. (2011). Access overlays: Improving nonvisual access to large touch screens for blind users. In Proceedings of the 24th annual 
ACM symposium on User interface software and technology - UIST '11 (pp. 273-282). New York, New York, USA: ACM Press.

Katz, B. F. G., Dramas, F., Parseihian, G. G. etan, Gutierrez, O., Kammoun, S., Brilhault, A., ... Denis, M. (2012). NAVIG: Guidance system for the visually impaired using virtual augmented reality. Technology and Disability, 24(2), 163-178.

Kelley, J. F. (1984). An iterative design methodology for user-friendly natural language office information applications. ACM Transactions on Information Systems, 2(1), 26-41.

Mereu, S. W., \& Kazman, R. (1996). Audio enhanced 3D interfaces for visually impaired users. In Proceedings of the SIGCHI conference on Human factors in computing systems common ground - $\mathrm{CHI}$ '96 (pp. 72-78) New York, New York, USA: ACM Press.

Pielot, M., Henze, N., \& Boll, S. (2009). Supporting map-based wayfinding with tactile cues. In Proceedings of the 11th International Conference on Human-Computer Interaction with Mobile Devices and Services MobileHCl '09. New York, New York, USA: ACM Press.

Pielot, M., Poppinga, B., Heuten, W., \& Boll, S. (2011). A tactile compass for eyes-free pedestrian navigation. In P. Campos, N. Graham, J. Jorge, N. Nunes, P. Palanque, \& M. Winckler (Eds.), Human-Computer Interaction - Interact 2011, LNCS 6947 (pp. 640-656). Lisbon, Portugal: Springer Berlin Heidelberg.

Roentgen, U. R., Gelderblom, G. J., Soede, M., \& de Witte, L. P. (2008). Inventory of Electronic Mobility Aids for Persons with Visual Impairments: A Literature Review. JVIB, 102(11), 702-724.
Sánchez, J., \& Hassler, T. (2006). AudioMUD: A multi-user virtual environment for blind people. In 2006 International Workshop on Virtual Rehabilitation (Vol. 15, pp. 64-71). IEEE.

Schloerb, D. W., Lahav, O., Desloge, J. G., \& Srinivasan, M. A. (2010). BlindAid: Virtual environment system for self-reliant trip planning and orientation and mobility training. In 2010 IEEE Haptics Symposium (pp. 363-370). IEEE.

Souman, J. L., Frissen, I., Sreenivasa, M. N., \& Ernst, M. O. (2009). Walking straight into circles. Current Biology: CB, 19(18), 1538-42.

Strothotte, T., Fritz, S., Michel, R., Raab, A., Petrie, H., \& Johnson, V. (1996). Development of dialogue systems for a mobility aid for blind people: initial design and usability testing. In international ACM SIGACCESS conference on Computers and accessibility (pp. 139-144).

Su, J., Rosenzweig, A., Goel, A., de Lara, E., \& Truong, K. N. (2010). Timbremap: : Enabling the Visually-Impaired to Use Maps on TouchEnabled Devices. In Proceedings of the 12th international conference on Human computer interaction with mobile devices and services - MobileHCl '10 (pp. 17-26). New York, New York, USA: ACM Press.

Tatham, A. F. (1991). The design of tactile maps: theoretical and practical considerations. In M. Rybaczak \& K. Blakemore (Eds.), Proceedings of international cartographic association: mapping the nations (pp. 157-166). London, UK: ICA.

Thorndyke, P. W., \& Hayes-Roth, B. (1982). Differences in spatial knowledge acquired from maps and navigation. Cognitive Psychology, 14(4), 560-589.
Tsukada, K., \& Yasumura, M. (2004). ActiveBelt: Belt-Type Wearable Tactile Display for Directional Navigation. In N. Davies, E. D. Mynatt, \& I. Siio (Eds.), UbiComp 2004: Ubiquitous Computing, LNCS Vol 3205 (Vol. 3205, pp. 384-399). Berlin, Heidelberg: Springer Berlin Heidelberg.

Walker, B. N., \& Lindsay, J. (2005). Navigation Performance in a Virtual Environment with Bonephones. In Proc. of the Int'l Conf. on Auditory Display (pp. 260-263).

Weber, B., Schatzle, S., Hulin, T., Preusche, C., \& Deml, B. (2011). Evaluation of a vibrotactile feedback device for spatial guidance. In 2011 IEEE World Haptics Conference (pp. 349-354). IEEE.

Williams, M. A., Hurst, A., \& Kane, S. K. (2013). "Pray before you step out": describing personal and situational blind navigation behaviors. In Proceedings of the 15th International ACM SIGACCESS Conference on Computers and Accessibility - ASSETS '13. New York, New York, USA: ACM Press.

Yairi, I. E., Takano, M., Shino, M., \& Kamata, M. (2008). Expression of paths and buildings for universal designed interactive map with due consideration for visually impaired people. In 2008 IEEE International Conference on Systems, Man and Cybernetics (pp. 524-529). IEEE.

Yatani, K., Banovic, N., \& Truong, K. (2012). SpaceSense: representing geographical information to visually impaired people using spatial tactile feedback. In Proceedings of the 2012 ACM annual conference on Human Factors in Computing Systems - CHI '12 (pp. 415-424). New York, New York, USA: ACM Press. 
1 Dr. Anke Brock is a research scientist at Inria Bordeaux, France. She obtained a PhD (2013) and Master in Human-Computer Interaction from the University of Toulouse, France, and an engineering diploma in Information Technology from Baden-Wuerttemberg Cooperative State University (Stuttgart, Germany). Her research interests include accessibility for visually impaired people, interactive geographic maps and advanced interaction techniques.

Webpage: www.ankebrock.com

E-Mail: anke.brock@inria.fr

2 Dr. Slim Kammoun obtained his Phd in computer science from Toulouse University (France) in 2013. Currently he is assistant professor at Tunis University, Tunisia, working in the Research Laboratory for Information and Communication Technologies \& Electrical Engineering (LaTICE) at the same university. His main research interests are assistive technology for the Blind with a focus on improving Mobility and Orientation for visually impaired pedestrians.

E-mail: slim.kammoun@esstt.rnu.tn

3 Dr. Marc Macé attended the University of Toulouse where he received a PhD degree in neurosciences in 2006 for his work on visual categorization in primates. After a postdoc in Leuven, Belgium, he joined the CNRS in France and is currently a researcher at the IRIT computer science institute in Toulouse. His main research topic is on assistive technologies for the Blind. Webpage: http://www.irit.fr/ Marc.Mace E-Mail: marc.mace@irit.fr.
4 Dr. Christophe Jouffrais is with the IRIT Lab (UMR5505, CNRS \& Univ of Toulouse) in Toulouse, FR. He is a CNRS researcher with a background in Cognitive Neuroscience and Assistive Technology. He holds a European PhD (2000) in Cognitive Neuroscience from the University of Lyon, FR and the University of Fribourg, CH. His current research focuses on blind human perception, action and cognition with an emphasis on non-visual human-computer interaction, and Assistive Technologies for the Blind. Webpage: http://www.irit.fr/ Christophe.Jouffrais/;

E-Mail: Christophe.jouffrais@irit.fr
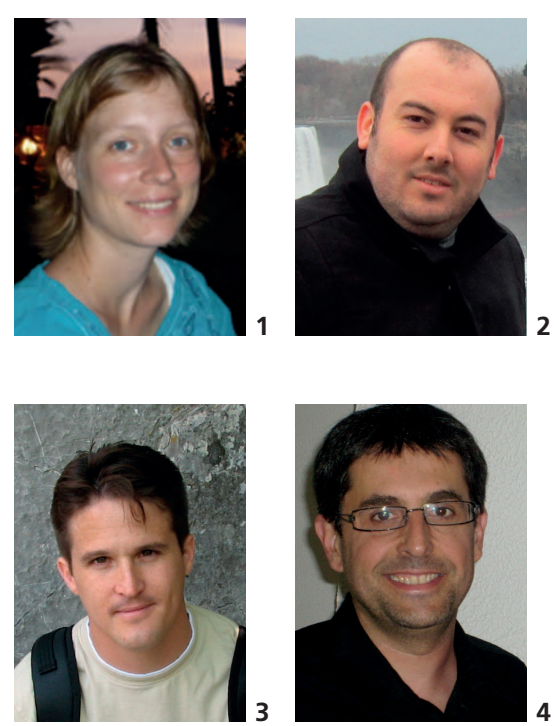